\title{
Uma Introdução à Teoria dos Jogos
}

\section{A Introduction to the Game Theory}

\author{
David Jonnes Francez \\ Universidade Federal de Santa Catarina, Floriánopolis, SC \\ davidfrancez@gmail.com
}

Resumo: A Teoria dos Jogos é um ramo da matemática aplicada que estuda situações estratégicas onde jogadores escolhem diferentes ações na tentativa de melhorar seu ganho. Inicialmente desenvolvida como ferramenta para compreender comportamento econômico, a teoria dos jogos é hoje usada em diversos campos acadêmicos. O objetivo do trabalho é mostrar, a partir de jogos simples, os conceitos de soma-zero, estratégias, matriz de ganhos, jogos estritamente e não estritamente determinados.

Palavras-chave: Teoria dos Jogos; Maximin; Minimax; matriz de ganhos; Soma-zero.

Abstract: The Game Theory is a branch of applied mathematics whose main concern is the study strategic situations where players choose different actions in an attempt to improve their payoffs. Initially developed as a tool to understand economic behavior, game theory is now used in many academic fields. The objective of this work is to show, from simple games, the concepts of zero-sum, strategies, matrix of payoffs, games strictly and nonstrictly determined.

Key words: Game Theory; Maximin; Minimax; payoffs; Sum-zero.

\section{Introdução}

A teoria dos jogos foi inicialmente desenvolvida como um modelo para analisar situações de conflito, na busca da racionalidade para embasar as decisões entre os agentes envolvidos.

Alguns matemáticos já fizeram estudos de jogos. Porém, nenhum deles deu continuidade às suas pesquisas para melhor fundamentá-las. Assim, esta teoria começou a se tornar realmente um foco de estudo na Matemática na década de 30 , ganhando um enfoque maior no ano de 1944.

Em 1944 a teoria dos jogos foi voltada, principalmente, a aplicações na área da Economia, porém, a complexidade de suas contribuições conseguem abranger muitas áreas pela diversidade de sua aplicabilidade.

Os jogos a serem considerados nestes estudos não são aqueles em que o resultado está sujeito exclusivamente ao fator sorte, no qual a atuação do jogador independe do resultado, como o jogo de dados, por exemplo. Tratam-se daqueles que são denominados jogos de estratégia, nos quais os sujeitos envolvidos criam uma sequência de tomadas de decisões e portanto passam a ter responsabilidade no resultado do jogo.

Partimos do pressuposto que os jogadores envolvidos sempre estarão em busca de vencer, ou seja, eles criarão estratégias que possam maximizar suas possibilidades, sempre tendo como objetivo a vitória. Salientamos que há a possibilidade de empate, mas quando um

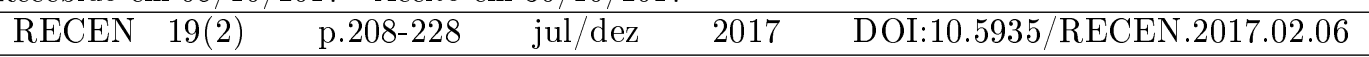


jogador vence, essa ação implica automaticamente na derrota de seu adversário, excluindo a possibilidade de ambos serem vencedores.

\section{Ideia de Jogo}

Nesta teoria, qualquer situação de conflito ou interação a ser considerada é denominada de "jogo", e os sujeitos envolvidos de "jogadores". Nesta pesquisa, por se tratar de uma introdução a esta complexa teoria, iremos limitar a utilização de situações em que constam apenas dois jogadores.

O exemplo a seguir serve para ilustrar a teoria dos jogos antes de introduzir a formalização matemática.

Exemplo 2.1 (Cobrança de Pênalti) Vamos supor agora que João e Pedro estejam fazendo uma disputa de pênaltis, no caso João é o goleiro e Pedro o cobrador, suponha ainda que Pedro possua apenas duas estratégias: chutar a bola no lado direito do gol ou chutar a bola no lado esquerdo do gol. Como a distância entre o gol e a marca do pênalti é muito curta, isto impossibilita o goleiro João de determinar de que lado Pedro chutará a bola. Assim, ele deve escolher para que lado pular sem saber qual será a direção do chute. Suponha também que, sempre que o goleiro adivinhar corretamente o lado do chute, ele é capaz de fazer a defesa em $80 \%$ das vezes caso o chute seja à direita e $60 \%$ à esquerda. $O$ batedor possui um tiro certeiro quando chuta no lado direito, mas não é tão bom quando chuta do lado esquerdo. Se Pedro chutar do lado direito do gol e o goleiro pular para o lado esquerdo, a bola entrará com $100 \%$ de certeza. Se o batedor chutar do lado esquerdo do gol e o goleiro pular para o lado direito, a bola entrará no gol com uma probabilidade de $50 \%$.

Este jogo não é um jogo simétrico, ou seja, existem grupos de estratégias diferentes para cada jogador. Se forem realizadas muitas cobranças, os jogadores não devem manter uma única estratégia (Estratégia Pura), pois colocaria seu oponente em vantagem. Nessas situações a melhor escolha é trocar constantemente de estratégia (Estratégia Mista). A vantagem da estratégia mista é que se coloca a dúvida na cabeça do oponente, pois ao alterar sistematicamente os lados para quais chuta, o cobrador não dá ao goleiro a certeza para qual lado chutará a bola. Da mesma forma, o chutador também não saberá exatamente qual canto escolher, se não tem certeza do que o goleiro fará.

Perceba que caso o goleiro pule para o mesmo lado que a bola, então terá $80 \%$ ou $60 \%$ de chance de fazer a defesa, dependendo do lado que Pedro chutar, gerando assim dois eventos favoráveis a João considerando os quatro possíveis (direita-direita e esquerdaesquerda). Enquanto Pedro estará certo do gol, caso faça a cobrança à direita e João pule para a esquerda. Por outro lado, caso Pedro cobre à esquerda e João pule à direita, isto não implica na certeza de gol, uma vez que o jogador não apresenta muita habilidade ao chutar para a esquerda, acertando assim, apenas a metade dos pênaltis. A matriz de ganhos da situação descrita fica assim:

Tabela 1. Cobrança de Pênalti

\begin{tabular}{ccc|c|} 
& \multicolumn{2}{c}{ João } \\
\multirow{2}{*}{ Pedro } & \multicolumn{2}{c}{ Direita } & \multicolumn{1}{c}{ Esquerda } \\
\cline { 3 - 4 } & Direita & $20 \%$ & $100 \%$ \\
\cline { 3 - 4 } & Esquerda & $50 \%$ & $40 \%$ \\
\cline { 3 - 4 } & & &
\end{tabular}


Revista Ciências Exatas e Naturais, Vol.19, nº.2, Jul/Dez, 2017

Pedro sabe que se chutar constantemente no lado esquerdo do gol, ele terá uma expectativa de converter no mínimo $40 \%$ das cobranças. Por outro lado, João percebe também tal informação e com o objetivo de minimizar as conversões de Pedro, escolhe sempre fazer defesas à esquerda, mantendo assim, suas chances em $60 \%$. Porém, como Pedro gosta muito de "blefar", decide trocar o lado da cobrança ocasionalmente. Sendo assim, vamos supor que ele faça a troca em $25 \%$ das vezes, João enfrentará o dilema de qual seria a melhor frequência para maximizar suas defesas.

Nesse exemplo, é extremamente importante não confundir taxa de sucesso com estratégia. Definiremos formalmente o que é estratégia mais a frente. Porém, para diferenciar essas ideias, entenda que estratégia é a frequência com que se escolhe uma opção em um jogo qualquer e taxa de sucesso é a probabilidade de ocorrência quando ambos os jogadores fazem suas respectivas escolhas.

Supondo que João queira minimizar as conversões de Pedro, e por isso decide trocar de lado metade das cobranças, a situação descrita pode ser resumida pela Tabela 2:

Tabela 2. Frequência Pênaltis I

\begin{tabular}{|c|c|c|c|c|}
\hline \multirow{4}{*}{$\begin{array}{c}\text { Frequência } \\
\text { Cobrança }\end{array}$} & \multirow{4}{*}{$25 \%$} & \multirow{4}{*}{$\begin{array}{l}\text { Direita } \\
\text { Esquerda }\end{array}$} & \multicolumn{2}{|c|}{$\begin{array}{c}\text { Frequência } \\
\text { Defesa }\end{array}$} \\
\hline & & & $\begin{array}{l}50 \% \\
\text { Direita }\end{array}$ & $\begin{array}{l}50 \% \\
\text { Esquerda }\end{array}$ \\
\hline & & & $20 \%$ & $100 \%$ \\
\hline & & & $50 \%$ & $40 \%$ \\
\hline
\end{tabular}

Analisando a Tabela 2 de frequências, podemos agora definir a probabilidade de cada um dos quatro eventos possíveis de ocorrerem. As respectivas chances de cada evento se encontram na Tabela 3 .

Tabela 3. Pênaltis I

\begin{tabular}{|l|l|l|}
\hline \multicolumn{1}{|c|}{$\begin{array}{c}\text { Evento } \\
\text { Composto }\end{array}$} & $\begin{array}{c}\text { Probabilidade } \\
\text { Evento }\end{array}$ & $\begin{array}{c}\text { Sucesso } \\
\text { Cobrança }\end{array}$ \\
\hline $\begin{array}{l}\text { Cobrar à direita e } \\
\text { defender à direita }\end{array}$ & $0,25.0,50=0,125$ & $20 \%$ \\
\hline $\begin{array}{l}\text { Cobrar à direita e } \\
\text { defender à esquerda }\end{array}$ & $0,25.0,50=0,125$ & $100 \%$ \\
\hline $\begin{array}{l}\text { Cobrar à esquerda e } \\
\text { defender à direita }\end{array}$ & $0,75.0,50=0,375$ & $50 \%$ \\
\hline $\begin{array}{l}\text { Cobrar à esquerda e } \\
\text { defender à esquerda }\end{array}$ & $0,75.0,50=0,375$ & $40 \%$ \\
\hline
\end{tabular}

Assim, podemos calcular a probabilidade de sucesso da cobrança de pênaltis em relação a Pedro:

$$
\begin{gathered}
0,125.20 \%+0,125.100 \%+0,375.50 \%+0,375.40 \%= \\
2,5 \%+12,5 \%+18,75 \%+15 \%=48,75 \%
\end{gathered}
$$

Note que devido ao blefe de Pedro, este conseguiu criar uma situação de sucesso de 
$48,75 \%$, o que gera um aumento de aproximadamente $22 \%$ em relação a manter as cobranças na esquerda, no qual garantiria em exatamente $40 \%$ suas chances de êxito.

Essa análise traz vários questionamentos que serão respondidos nos próximos capítulos: Pedro pode aumentar suas chances para além de $48,5 \%$ com uma estratégia diferente? Qual é a maior probabilidade de sucesso que Pedro pode fazer? Qual a melhor resposta de João para qualquer estratégia de Pedro? João consegue definir uma melhor estratégia independente das decisões de Pedro? As respostas serão respondidas mais adiante.

\section{Estratégia}

A teoria dos jogos pode ser definida como a teoria dos modelos matemáticos que estuda a escolha de decisões ótimas sob condições de conflitos. O elemento básico em um jogo é o conjunto de jogadores que dele participam e suas estratégias. Neste trabalho definiremos estratégia como o conjunto das frequências com que um jogador escolhe as opções de um determinado jogo. Assim, diante de suas escolhas, cria-se uma situação ou perfil no espaço de todas as situações (perfis) possíveis.

Os detalhes específicos de cada jogo serão ignorados no exemplo subsequente e simplesmente trataremos de uma matriz de números, sem unidade de medida. Portanto, podemos tratar um jogo de soma-zero $2 \times 2$ com a forma:

Tabela 4. Soma-Zero: Genérico

\begin{tabular}{c|c|c|}
\multicolumn{1}{c}{ João } \\
\cline { 2 - 3 } Pedro & $a_{11}$ & $a_{12}$ \\
\cline { 2 - 3 } & $a_{21}$ & $a_{22}$ \\
\cline { 2 - 3 } & &
\end{tabular}

no qual $a_{11}, a_{12}, a_{21}$ e $a_{22}$ são números quaisquer. Essa abstração matemática tem o intuito de simplificar cada jogo estudado, facilitando a compreensão dos mesmos. Entretanto, faremos discussões de alguns casos concretos para que os exemplos se tornem palpáveis e de fácil entendimento.

Cada jogo de soma-zero $2 \times 2$ tem dois jogadores, que continuaremos chamando de Pedro e João. Quando os jogadores tomam alguma decisão, consequentemente, escolhem uma linha ou uma coluna da matriz. Especificamente nos exemplos anteriores, Pedro escolhe uma linha e João uma coluna. Por exemplo, suponha que no jogo Cobrança de Pênalti Pedro chute à direita e João pule à esquerda, a situação descrita seria assim:

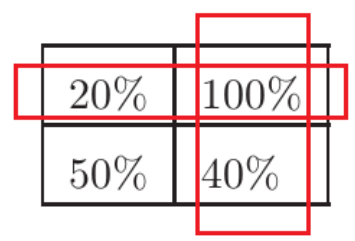

Figura 1. Tomada de Decisão

Cada jogador faz sua escolha simultânea e independentemente do oponente. Assim, a seleção da linha e coluna representa o resultado da jogada, e a entrada da matriz, cujo elemento está contido na intersecção da linha e coluna selecionada, é o ganho do jogador 
Revista Ciências Exatas e Naturais, Vol.19, nº.2, Jul/Dez, 2017

(payoff). No caso acima o ganho de Pedro é 100\%. Por outro lado, se Pedro tivesse chutado à esquerda e João mantido sua escolha, o ganho do batedor seria de $40 \%$. Note que o ganho é em relação ao cobrador, conforme comentado no capítulo anterior, e obviamente o ganho de Pedro implica diretamente a perda de João.

Como o objetivo de Pedro é maximizar suas chances de gol e João de minimizar o sucesso das cobranças, cada jogador aplica uma estratégia, ou seja, toma uma decisão entre as opções possíveis.

Definição 3.1 Uma estratégia em um jogo de soma-zero $2 \times 2$ é um par de números $p_{1}, p_{2}$, denotaremos $\left[p_{1} ; p_{2}\right]$, em que:

$$
0 \leqslant p_{1} \leqslant 1, \quad 0 \leqslant p_{2} \leqslant 1 \quad \text { onde } p_{1}+p_{2}=1
$$

no qual $p_{1}$ representa a frequência com que a primeira linha (ou coluna) é escolhida, e $p_{2}$ a frequência com que a segunda linha (ou coluna) é escolhida.

No Exemplo Cobrança de Pênalti Pedro faz a escolha de chutar $25 \%$ das vezes para o lado direito, ou seja, o par de números que representa sua estratégia é $[0,25 ; 0,75]$, enquanto a estratégia inicial de João é manter metade de suas defesas para cada lado, ou seja, sua estratégia é $[0,5 ; 0,5]$. Quando se faz a discussão de estratégias de um modo geral, pode-se definir a estratégia de Pedro como $[1-p ; p]$ e a de João como $[1-q ; q]$.

Conforme visto anteriormente no jogo Cobrança de Pênalti, cada jogador aplicou uma estratégia e com isso foi possível estabelecer a probabilidade de ganho para cada oponente. Agora será feito o cálculo de maneira genérica em termos de um jogo de soma-zero $2 \times 2$, tomando as estratégias $[1-p ; p][q ; 1-q]$ obteremos a seguinte matriz:

Tabela 5. Soma-Zero:Genérico II

\begin{tabular}{l|l|l|}
\multicolumn{1}{c}{} & \multicolumn{1}{l}{$\mathbf{1 - q}$} & \multicolumn{1}{l}{$\mathbf{q}$} \\
\cline { 3 - 3 } $\mathbf{1} \mathbf{1} \mathbf{p}$ & $a_{11}$ & $a_{12}$ \\
\cline { 2 - 3 } $\mathbf{p}$ & $a_{21}$ & $a_{22}$ \\
\cline { 2 - 3 } & &
\end{tabular}

Vamos supor que Pedro tenha escolhido uma linha e João uma coluna. Desde que ambos os jogadores tenham feito suas escolhas de maneiras independentes, podemos concluir que:

- A probabilidade de Pedro receber o ganho $a_{11}$ é $(1-p) \times(1-q)$

- A probabilidade de Pedro receber o ganho $a_{12}(1-p) \times q$

- A probabilidade de Pedro receber o ganho $a_{21} p \times(1-q)$

- A probabilidade de Pedro receber o ganho $a_{22} p \times q$

E como os quatro eventos são mutuamente exclusivos e cobrem todas as possibilidades, segue que o ganho esperado de Pedro $\left(G_{\mathcal{P}}\right)$ é uma função de variáveis $p$ e $q$ definida por:

$$
G_{\mathcal{P}}(p, q)=(1-p) \cdot(1-q) \cdot a_{11}+(1-p) \cdot q \cdot a_{12}+p \cdot(1-q) \cdot a_{21}+p \cdot q \cdot a_{22} .
$$

O diagrama representado pela Tabela 14 serve de auxílio para o cálculo dos ganhos de cada jogador. Tais diagramas são chamados de diagramas auxiliares, com eles pode-se computar de forma rápida e precisa o resultado do jogo, principalmente quando aplicado em uma rotina repetidas vezes. 
FRANCEZ, D. J.

Exemplo 3.1 No jogo Cobrança de Pênalti, supondo que Pedro tenha uma estratégia $[0,3 ; 0,7]$ e João $[0,6 ; 0,4]$, então o diagrama auxiliar seria :

Tabela 6. Exemplo 3.1

\begin{tabular}{|c|c|c|}
\hline & $60 \%$ & $40 \%$ \\
\hline $30 \%$ & $20 \%$ & $100 \%$ \\
\hline $0 \%$ & $50 \%$ & $40 \%$ \\
\hline
\end{tabular}

Tomando os cálculos dos ganhos de Pedro, temos

$$
\begin{gathered}
0,3.0,6.20 \%+0,3.0,4.100 \%+0,7.0,6.50 \%+0,7.0,4.40 \% \\
3,6 \%+12 \%+21 \%+11,2 \%=47,8 \%
\end{gathered}
$$

Portanto, quando Pedro e João aplicarem, respectivamente, as estratégias $[0,3 ; 0,7]$ e $[0,6 ; 0,4]$ então, Pedro deve esperar um sucesso em $47,8 \%$ de suas cobranças.

As estratégias de um jogador podem ser classificadas de duas maneiras: Estratégias Puras e Estratégias Mistas. Uma estratégia é dita pura quando o jogador escolhe apenas uma das opções do jogo, ou seja, a frequência de uma determinada opção é 1, enquanto todas as outras é nula.

Definição 3.2 Dada uma estratégia $p_{1}, p_{2}, p_{3}, \ldots p_{m}$ em um jogo de soma-zero $m \times n$, tal estratégia é dita pura quando existir $p_{k}=1$ para algum $k \in(1,2,3, \ldots m)$.

Por outro lado, uma estratégia é dita mista quando não for pura, ou seja, o jogador não escolhe constantemente uma única opção do jogo.

Definição 3.3 Dada uma estratégia $p_{1}, p_{2}, p_{3}, \ldots p_{m}$ em um jogo de soma-zero $m \times n$, tal estratégia é dita mista quando $p_{k} \neq 1$ para todo $k \in(1,2,3, \ldots m)$.

No jogo Cobrança de Pênalti, Pedro possui duas estratégias puras: $[1,0]$ e $[0,1]$ que representariam, respectivamente, cobranças de pênaltis apenas à direita e apenas à esquerda. Estratégias que não são puras são chamadas de mistas. Portanto, $[0,1 ; 0,9]$ e $[1-p ; p]$ são estratégias mistas, contanto é claro, que $p$ não seja 0 ou 1.

Ao final desse capítulo, seria natural nos perguntarmos: qual a melhor estratégia possível para maximizar os ganhos de um jogador? E como poríamos encontrá-la? A resposta será discutida a seguir.

\section{Respostas Ideais para Estratégias Específicas}

Nesse capítulo procuraremos encontrar as melhores táticas quando sabe-se a estratégia do oponente. Antes de fazermos essa análise, devemos ter precaução, pois deduzir a estratégia do adversário a partir de movimentos anteriores não é uma tarefa simples, e por mais que encontremos tal estratégia, isto não significa que saberemos a próxima jogada do oponente. 
Revista Ciências Exatas e Naturais, Vol.19, nº.2, Jul/Dez, 2017

Uma estratégia é uma lista de números que representa a frequência com que cada opção é escolhida no jogo.

Voltemos ao jogo Cobranças de Pênalti. Em dado momento Pedro definiu a estratégia $[0,25 ; 0,75]$, ou seja, cobrar $25 \%$ das vezes à direita e $75 \%$ à esquerda. Suponha que João perceba a estratégia de seu adversário e procure a melhor resposta para minimizar o sucesso de Pedro. Seja $[1-q ; q]$ a melhor estratégia para João, portanto o diagrama auxiliar seria dado por:

Tabela 7. Resposta Ideal Pênalti I

\begin{tabular}{|c|c|c|}
\hline & $1-q$ & $q$ \\
\hline $25 \%$ & $20 \%$ & $100 \%$ \\
\hline & $50 \%$ & $40 \%$ \\
\hline
\end{tabular}

e a expectativa de Pedro é

$$
\begin{aligned}
& 0,25 \cdot(1-q) \cdot 0,2+0,25 \cdot q \cdot 1+0,75 \cdot(1-q) \cdot 0,5+0,75 \cdot q \cdot 0,4 \\
& =0,05(1-q)+0,25 q+0,375(1-q)+0,3 q \\
& =0,05-0,05 q+0,25 q+0,375-0,375 q+0,3 q \\
& =0,425-0,125 q
\end{aligned}
$$

Perceba então, que o ganho de Pedro depende da estratégia a ser aplicada por João, ou seja, o payoff do cobrador está em função da frequência $q$ do goleiro. Note que o menor valor do ganho de Pedro acontece quando João decide defender exclusivamente à esquerda, ou seja, tomando $q=1$ entre todos os valores possíveis de $q$, assim o ganho seria dado por:

$$
0,425-0,125 q=0,425-0,125.1=0,3
$$

Portanto, o ganho esperado de Pedro é $30 \%$, consequentemente, João tem em média $70 \%$ de sucesso.

\begin{tabular}{|c|c|c|}
\hline & $1-q$ & $q$ \\
\hline $50 \%$ & $20 \%$ & $100 \%$ \\
\hline $5 \%$ & $50 \%$ & $40 \%$ \\
\hline
\end{tabular}

Suponha agora que Pedro diversificará seus chutes à direita e à esquerda igualmente, ou seja, aplicará a estratégia $[0,5 ; 0,5]$. Qual seria a resposta ideal de João para minimizar o sucesso de Pedro? Vejamos o diagrama auxiliar

Tabela 8. Resposta Ideal Pênalti II

e portanto o ganho esperado é

$$
\begin{aligned}
& 0,5 \cdot(1-q) \cdot 0,2+0,5 \cdot q \cdot 1+0,5 \cdot(1-q) \cdot 0,5+0,5 \cdot q \cdot 0,4 \\
& =0,1(1-q)+0,5 q+0,25(1-q)+0,2 q \\
& =0,1-0,1 q+0,5 q+0,25-0,25 q+0,2 q \\
& =0,35+0,35 q .
\end{aligned}
$$

Analisando a expressão com o intuito de minimizá-la, basta João tomar a contra-estratégia $[1 ; 0]$ para que o sucesso do cobrador seja reduzido a $35 \%$. Entre outras palavras, caso Pedro 
chute metade dos pênaltis à direita e a outra metade à esquerda, a melhor escolha para o goleiro seria apostar constantemente no lado direito.

Quando um jogador aplica uma estratégia qualquer, seu oponente procura uma tática que resulte no ganho mínimo de seu adversário, tal tática chamaremos de contra-estratégia ideal. Note que nos exemplos anteriores, as duas contra-estratégias de João são puras, isto de certa forma não é mera coincidência. Podemos formular esse princípio de maneira mais geral, como um teorema.

Teorema 4.1 Se um dos dos jogadores aplicar uma estratégia fixa, então seu oponente tem uma contra-estratégia ideal e ela é pura.

Demonstração: Suponha que João aplique uma estratégia fixa $\left[1-q_{0} ; q_{0}\right]$ e Pedro deseja encontrar a contra-estratégia ideal, ou seja, procura maximizar seus ganhos. Vejamos a situação descrita em um jogo genérico abaixo:

Tabela 9. Demonstração 4.1

\section{João}

\begin{tabular}{|c|c|c|c|}
\hline \multirow{3}{*}{ Pedro } & \multirow{3}{*}{$1-p$} & $1-q_{0}$ & $q_{0}$ \\
\hline & & $a_{11}$ & $a_{12}$ \\
\hline & & $a_{21}$ & $a_{22}$ \\
\hline
\end{tabular}

Note então que a função ganho de Pedro é dada por:

$$
G_{\mathcal{P}}(p)=(1-p) \cdot\left(1-q_{0}\right) \cdot a_{11}+(1-p) \cdot q_{0} \cdot a_{12}+p \cdot\left(1-q_{0}\right) \cdot a_{21}+p \cdot q_{0} \cdot a_{22}
$$

fazendo as operações de multiplicação e colocando $p$ em evidência temos:

$$
G_{\mathcal{P}}(p)=p\left(q_{0}\left(a_{11}-a_{12}-a_{21}+a_{22}\right)-a_{11}+a_{21}\right)+a_{11}+q_{0} \cdot\left(a_{12}-a_{11}\right)
$$

Observe que $G_{\mathcal{P}}$ é uma função do tipo afim com variável independente $p$, logo seu gráfico é representado por um segmento de reta,uma vez que a função está definida apenas para $0 \leqslant p \leqslant 1$. Assim, o ponto de máximo é dado pela extremidade do segmento, ou seja, para $p=0$ ou $p=1$, portanto a contra-estratégia ideal para Pedro é dada por $[0 ; 1]$ ou $[1 ; 0]$ e ambas são puras como queríamos mostrar.

\section{A estratégia Maximin}

Neste capítulo buscaremos uma estratégia ideal, ou seja, procurar a melhor tática possível para Pedro em qualquer jogo de soma-zero $2 \times 2$, justificando-a matematicamente. Seja o jogo:

Tabela 10. Estratégia Ideal I

\begin{tabular}{c|c|c|}
\multicolumn{2}{c}{ João } \\
\cline { 2 - 3 } Pedro $a_{11}$ & $a_{12}$ \\
\cline { 2 - 3 }$a_{21}$ & $a_{22}$ \\
\cline { 2 - 3 } & &
\end{tabular}


Revista Ciências Exatas e Naturais, Vol.19, nº.2, Jul/Dez, 2017

Devido ao Teorema 3.1, sabemos que para qualquer estratégia $[1-p, p]$ imposta por Pedro, existe uma contra-estratégia pura que João pode aplicar para minimizar o ganho de seu adversário. Sejam $g_{1}(p)$ e $g_{2}(p)$ os respectivos ganhos de Pedro quando o mesmo aplica as estratégias puras $[1,0]$ e $[0,1]$. Segue abaixo os diagramas auxiliares da situação descrita:

Tabela 11. Estratégia Ideal II

\begin{tabular}{l|l|l|}
\multicolumn{1}{c}{} & \multicolumn{1}{l}{1} & \multicolumn{1}{l}{0} \\
\cline { 2 - 3 } $1-\mathrm{p}$ & $a_{11}$ & $a_{12}$ \\
\cline { 2 - 3 } $\mathrm{p}$ & $a_{21}$ & $a_{22}$ \\
\cline { 2 - 3 } & &
\end{tabular}

Tabela 12. Estratégia Ideal III

\begin{tabular}{l|l|l|}
\multicolumn{1}{c}{} & \multicolumn{1}{l}{0} & \multicolumn{1}{l}{1} \\
\cline { 2 - 3 } 1-p & $a_{11}$ & $a_{12}$ \\
\cline { 2 - 3 } $\mathrm{p}$ & $a_{21}$ & $a_{22}$ \\
\cline { 2 - 3 } & &
\end{tabular}

portanto o ganho é dado por

$$
\begin{aligned}
& g_{1}(p)=(1-p) \cdot 1 \cdot a_{11}+p \cdot 1 \cdot a_{21}=a_{11}(1-p)+a_{21} p=\left(a_{21}-a_{11}\right) p+a_{11} . \\
& g_{2}(p)=(1-p) \cdot 1 \cdot a_{12}+p \cdot 1 \cdot a_{22}=a_{12}(1-p)+a_{22} p=\left(a_{22}-a_{12}\right) p+a_{12} .
\end{aligned}
$$

Se denotarmos como $G_{P}(p)$ a expectativa do ganho de Pedro aplicando as estratégias descritas acima, mesmo sabendo que João pode minimizá-las, então o ganho de Pedro é dado por

$$
G_{P}(p)=\min \left(g_{1}(p), g_{2}(p)\right)
$$

Acabamos de determinar o ganho de Pedro $G_{\mathcal{P}}(p)$ em função de $p$, ou seja, temos uma relação direta entre a estratégia $[1-p ; p]$ aplicada e o ganho esperado.

Perceba que a variável independente $p$ aparece nas expressões $g_{1}(p)$ e $g_{2}(p)$ como uma função afim, portanto, o respectivo gráfico é dado por uma reta. Perceba, é claro, que o valor de $p$ é um número real entre 0 e 1 , uma vez que $p$ representa uma probabilidade, assim, o gráfico da função descrita é formado por segmentos de reta no intervalo $[0,1]$. Note que as extremidades dessa função são dadas por:

$$
\begin{aligned}
& g_{1}(0)=\left(a_{21}-a_{11}\right) \cdot 0+a_{11}=a_{11} . \\
& g_{1}(1)=\left(a_{21}-a_{11}\right) \cdot 1+a_{11}=a_{21} .
\end{aligned}
$$

Isso mostra que o gráfico de $g_{1}(p)$ é um segmento de reta que contém as extremidades $\left(0, a_{11}\right)$ e $\left(1, a_{21}\right)$. Analogamente:

$$
\begin{aligned}
& g_{2}(0)=\left(a_{22}-a_{12}\right) \cdot 0+a_{12}=a_{12} . \\
& g_{2}(1)=\left(a_{22}-a_{12}\right) \cdot 1+a_{12}=a_{22} .
\end{aligned}
$$

e o gráfico de $g_{2}(p)$ é um segmento de reta que contém as extremidades $\left(0, a_{12}\right)$ e $\left(1, a_{22}\right)$. Com essas informações de $g_{1}(p)$ e $g_{2}(p)$, podemos notar que ao aplicar a estratégia $[1-p ; p]$ ou $[p ; 1-p]$, consequentemente, as funções teriam seus gráficos trocados, ou seja, o gráfico de $g_{1}(p)$ seria de $g_{2}(p)$ e vice-versa. O gráfico de $G_{\mathcal{P}}(p)$ é feito a partir da observação seguinte:

O gráfico de $G_{\mathcal{P}}(p)$ consiste em uma linha que, para qualquer valor possivel de $p$, contém o menor valor entre $g_{1}(p)$ e $g_{2}(p)$. 
O exemplo a seguir mostram uma variedade de informações importantes para a escolha de uma estratégia, apenas observando o gráfico da função.

Exemplo 5.1 Para o jogo do Cobranças de Pênalti tínhamos a seguinte matriz de ganhos:

Tabela 13. Exemplo 2.1 - Cobrança de Pênalti

\begin{tabular}{|l|l|}
\hline $20 \%$ & $100 \%$ \\
\hline $50 \%$ & $40 \%$ \\
\hline
\end{tabular}

Temos $a_{11}=0,2, a_{12}=1, a_{21}=0,5$ e $a_{22}=0,4$. Determinando n $g_{1}(p)$ e $g_{2}(p)$, temos:

$$
\begin{aligned}
& g_{1}(p)=\left(a_{21}-a_{11}\right) p+a_{11}=(0,5-0,2) p+0,2=0,3 p+0,2 . \\
& g_{2}(p)=\left(a_{22}-a_{12}\right) p+a_{12}=(0,4-1) p+1=-0,6 p+1 .
\end{aligned}
$$

e o gráfico da função é dada pela Figura 5 abaixo:

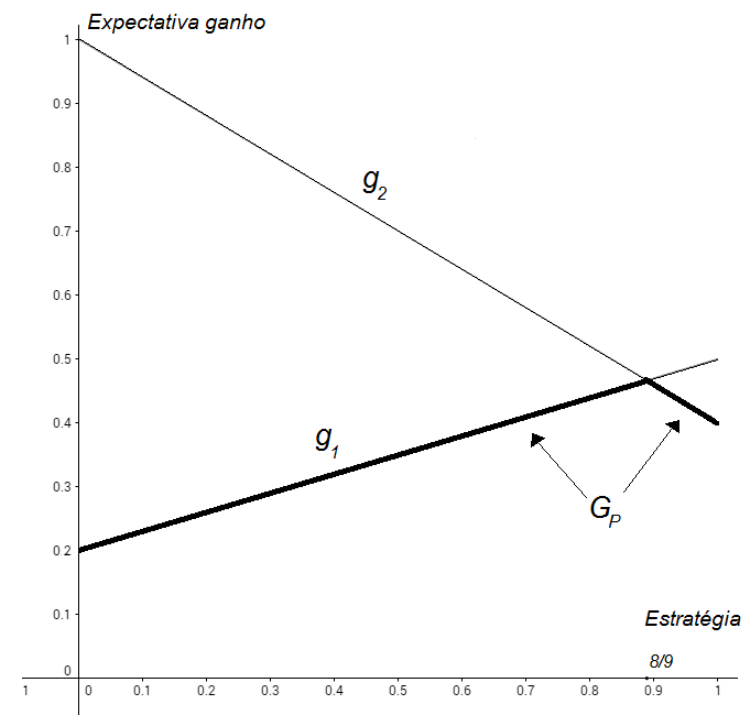

Figura 2. Exemplo 5.1 - Gráfico Expectativa

Perceba que o gráfico de $G_{\mathcal{P}}$ é dado grande parte por $g_{1}(p)$, onde João aplicaria a contra-estratégia pura $[1 ; 0]$ até o determinado momento em que João trocaria de estratégia e passaria a aplicar $[0 ; 1]$. Note que o momento de troca de estratégia é exatamente a intersecção de $g_{1}(p)$ e $g_{2}(p)$. Esse ponto é de extrema importância para a análise do jogo, podemos encontrá-lo facilmente resolvendo a equação:

$$
g_{1}(p)=g_{2}(p)
$$

isto é, 
Revista Ciências Exatas e Naturais, Vol.19, nº.2, Jul/Dez, 2017

$$
\begin{aligned}
0,3 p+0,2 & =-0,6 p+1 \\
0,9 p & =0,8 \\
p & =\frac{8}{9} .
\end{aligned}
$$

Consequentemente,

$$
\begin{aligned}
& {[1 ; 0] \text { é uma contra-estratégia ideal para João quando } 0 \leqslant p \leqslant \frac{8}{9} .} \\
& {[0 ; 1] \text { é uma contra-estratégia ideal para João quando } \frac{8}{9} \leqslant p \leqslant 1}
\end{aligned}
$$

Fazendo uma análise informal, podemos concluir que caso Pedro chute à esquerda com uma frequência menor que $\frac{8}{9}$, então João irá persistir em defender à direita. Porém, quando Pedro faz as cobranças com probabilidade superior a $\frac{8}{9}$ à esquerda, então o goleiro fará suas defesas constantemente à esquerda. Finalmente, quando $p=\frac{8}{9}$ temos o mesmo valor da função independente da estratégia pura aplicada por João, ou seja, o ganho será o mesmo para $g_{1}(p)$ ou $g_{2}(p)$.

Uma vez que a intersecção dos gráficos de $g_{1}(p)$ e $g_{2}(p)$ é o ponto de máximo da função $G_{P}$ temos que esse ponto representa a escolha ideal para Pedro, assim temos que a estratégia é dada por:

$$
\left[1-\frac{8}{9} ; \frac{8}{9}\right]=\left[\frac{1}{9} ; \frac{8}{9}\right]
$$

Portanto, a maior expectativa de ganho que Pedro, levando em conta que João quer minimizá-la, pode garantir é calculada abaixo:

$$
g_{1}\left(\frac{8}{9}\right)=0,3 \cdot \frac{8}{9}+0,2=\frac{8}{30}+0,2=0,26 \ldots+0,2=0,26 \ldots=46,6 \ldots \%
$$

$\mathrm{ou}$

$$
g_{2}\left(\frac{8}{9}\right)=-0,6 \cdot \frac{8}{9}+1=-\frac{8}{15}+1=-0,53 \ldots+1=0.46 \ldots=46,66 \ldots \%
$$

Está claro nos exemplos vistos anteriormente, que o ponto de máximo da função $G_{P}$ é uma estratégia especial. Infelizmente, há situações nas quais há mais de um ponto máximo e devemos ter cautela. Porém podemos formular a seguinte definição/teorema:

Teorema 5.1 Se $(x, y)$ é o ponto de máximo do gráfico de $G_{\mathcal{P}}$ então:

$[1-x ; x]$ é uma estratégia Maximin para Pedro, e y é o ganho Maximin esperado.

Se Pedro aplicar a estratégia Maximin $[1-x ; x]$ então ele pode esperar vencer no minimo y em cada jogada. 
Essa nomenclatura é dada porque cada ponto do gráfico é o mínimo de duas possíveis escolhas de João, e por outro lado, Pedro está escolhendo o ponto mais alto do gráfico, ou seja, está maximizando o mínimo. Entre outras palavras, Pedro está escolhendo o valor mais alto abaixo da curva de mínimo.

Para concluir esse capítulo, percebemos que a estratégia Maximin é boa no sentido de garantir o ganho mínimo por jogada, além disso, é o melhor valor que pode ser assegurado. No entanto, é natural nos questionarmos se a estratégia Maximin é realmente a melhor tática para aumentarmos o ganho esperado. A resposta dessa questão, é claro, depende das circunstâncias de cada jogo.

\section{A Estratégia Minimax}

Vamos, agora, em busca de uma boa estratégia para João. O gráfico da expectativa de ganho de João em um jogo de soma-zero $2 \times 2$, pode ser encontrado de maneira análoga a de Pedro, porém, com algumas pequenas diferenças e similaridades que serão descritas abaixo.

Devido ao Teorema 4.1, sabemos que para qualquer estratégia $[1-q, q]$ aplicada por João em um jogo qualquer de soma-zero $2 \times 2$

Tabela 14. Estratégia Ideal IV

$$
\begin{array}{c|c|c|}
\multicolumn{2}{c}{\text { João }} \\
\cline { 2 - 3 } \text { Pedro } & \multicolumn{1}{c}{a_{11}} & a_{12} \\
\cline { 2 - 3 } & a_{21} & a_{22} \\
\cline { 2 - 3 } & &
\end{array}
$$

existe uma contra-estratégia pura que Pedro pode aplicar para maximizar seu ganho. Sejam $h_{1}(q)$ e $h_{2}(q)$ os respectivos ganhos de Pedro quando o mesmo aplica as estratégias puras $[1 ; 0]$ e $[0 ; 1]$. Seguem abaixo os diagramas auxiliares da situação descrita:

Tabela 15. Estratégia Ideal VI

\begin{tabular}{l|l|l|}
\multicolumn{1}{c}{$1-\mathrm{q}$} & \multicolumn{1}{c}{$\mathrm{q}$} \\
\cline { 2 - 3 } 1 & $a_{11}$ & $a_{12}$ \\
\cline { 2 - 3 } 0 & $a_{21}$ & $a_{22}$ \\
\cline { 2 - 3 } & &
\end{tabular}

Tabela 16. Estratégia Ideal VII

\begin{tabular}{l|l|l|}
\multicolumn{1}{c}{$1-\mathrm{q}$} & \multicolumn{1}{c}{$\mathrm{q}$} \\
\cline { 2 - 3 } 0 & $a_{11}$ & $a_{12}$ \\
\cline { 2 - 3 } 1 & $a_{21}$ & $a_{22}$ \\
\cline { 2 - 3 } & &
\end{tabular}

portanto o ganho de Pedro é dado por

$$
\begin{aligned}
& h_{1}(q)=(1-q) \cdot 1 \cdot a_{11}+q \cdot 1 \cdot a_{12}=a_{11}(1-q)+a_{12} q=\left(a_{12}-a_{11}\right) q+a_{11} . \\
& h_{2}(q)=(1-q) \cdot 1 \cdot a_{21}+q \cdot 1 \cdot a_{22}=a_{21}(1-q)+a_{22} q=\left(a_{22}-a_{21}\right) q+a_{21} .
\end{aligned}
$$

Se denotarmos como $G_{\mathcal{J}}(q)$ a expectativa do ganho de Pedro aplicando as estratégias descritas acima, mesmo sabendo que ele pode maximizá-las, então o ganho de Pedro é dado por:

$$
G_{\mathcal{J}}(q)=\max \left(h_{1}(q), h_{2}(q)\right)
$$

Acabamos de determinar o ganho de Pedro $G_{J}(q)$ em função de $q$, ou seja, temos uma relação direta entre a estratégia $[1-q ; q]$ aplicada e o ganho esperado. 
Revista Ciências Exatas e Naturais, Vol.19, nº.2, Jul/Dez, 2017

Perceba que a variável independente $q$ aparece nas expressões $h_{1}(q)$ e $h_{2}(q)$ como uma função afim, portanto o respectivo gráfico é dado por uma reta. Perceba é claro, que o valor de $q$ é um número real entre 0 e 1 , uma vez que $q$ representa uma probabilidade, assim o gráfico da função descrita é formado por segmentos de reta no intervalo $[0 ; 1]$. Note que as extremidades dessa função são dadas por:

$$
\begin{aligned}
& h_{1}(0)=\left(a_{12}-a_{11}\right) \cdot 0+a_{11}=a_{11} . \\
& h_{1}(1)=\left(a_{12}-a_{11}\right) \cdot 1+a_{11}=a_{12} .
\end{aligned}
$$

Isso mostra que o gráfico de $h_{1}(q)$ é um segmento de reta que contém as extremidades $\left(0, a_{11}\right)$ e $\left(1, a_{12}\right)$. Analogamente,

$$
\begin{aligned}
& h_{2}(0)=\left(a_{22}-a_{21}\right) \cdot 0+a_{21}=a_{21} . \\
& h_{2}(1)=\left(a_{22}-a_{21}\right) \cdot 1+a_{21}=a_{22} .
\end{aligned}
$$

e o gráfico de $h_{2}(q)$ é um segmento de reta que contém as extremidades $\left(0, a_{11}\right)$ e $\left(1, a_{12}\right)$. Com essas informações de $h_{1}(q)$ e $h_{2}(q)$ podemos notar que aplicar a estratégia $[1-q ; q]$ ou $[q ; 1-q]$ não mudaria o ganho esperado de João, pois essa escolha de estratégia implicaria em apenas uma troca de gráficos, ou seja, o gráfico de $h_{1}(q)$ seria de $h_{2}(q)$ e vice-versa. O gráfico de $G_{\mathcal{J}}(q)$ é feito a partir da observação seguinte:

$O$ gráfico de $G_{\mathcal{J}}(q)$ consiste em uma linha que, para qualquer valor possivel de q, contém o maior valor entre $h_{1}(q)$ e $h_{2}(q)$.

Agora reexaminaremos os exemplos anteriores visto sob a perspectiva de João.

Exemplo 6.1 No jogo Cobranças de Pênalti temos a seguinte matriz de ganhos:

Tabela 17. Exemplo 2.1 - Cobrança de Pênalti

\begin{tabular}{|l|l|}
\hline $20 \%$ & $100 \%$ \\
\hline $50 \%$ & $40 \%$ \\
\hline
\end{tabular}

temos então $a_{11}=0,2, a_{12}=1, a_{21}=0,5$ e $a_{22}=0,4$. Calculando as expressões de $h_{1}(q)$ e $h_{2}(q)$, temos:

$$
\begin{aligned}
& h_{1}(q)=\left(a_{12}-a_{11}\right) q+a_{11}=(1-0,2) q+0,2=0,8 q+0,2 . \\
& h_{2}(q)=\left(a_{22}-a_{21}\right) q+a_{21}=(0,4-0,5) q+0,5=-0,1 q+0,5 .
\end{aligned}
$$

e o gráfico da função é dada pela Figura 10 abaixo. 
FRANCEZ, D. J.

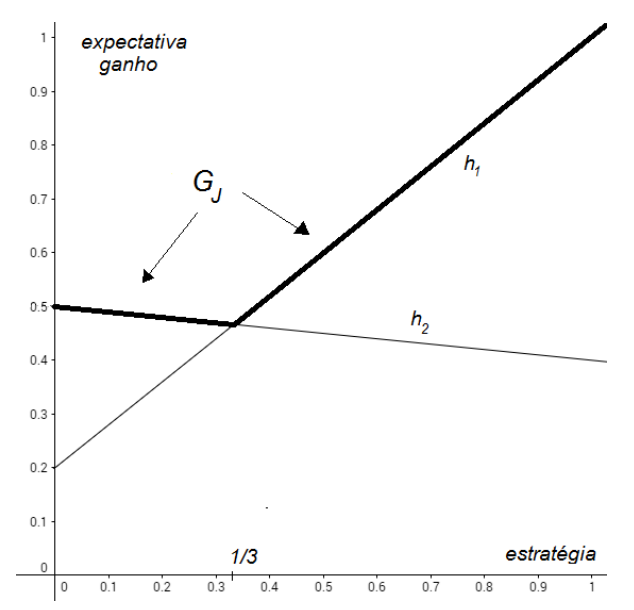

Figura 3. Exemplo 6.1 - Gráfico Expectativa

$\mathrm{O}$ gráfico de $G_{\mathcal{J}}$ coincide inicialmente com $h_{2}$ para pequenos valores de $q$. Portanto, quando João aplicar a estratégia $[1-q ; q]$ para valores próximos de zero, Pedro deve responder com a estratégia pura $[0 ; 1]$; quando $q$ está próximo de 1 , então Pedro emprega $[1 ; 0]$. De maneira mais informal, se João raramente defender à esquerda, Pedro irá cobrar exatamente nesse lado; se João pular à esquerda, então Pedro sempre chutará à direita. O ponto mais interessante para minimizar a expectativa de ganho de Pedro é a intersecção de $h_{1}(q)$ e $h_{2}(q)$. Para encontrar tal ponto basta resolvermos a equação abaixo:

$$
\begin{aligned}
h_{1}(q) & =h_{2}(q) \\
0,8 q+0,2 & =-0,1 q+0,5 \\
0,9 q & =0,3 \\
q & =\frac{1}{3} .
\end{aligned}
$$

Consequentemente,

$$
\begin{aligned}
& {[0 ; 1] \text { é uma contra-estratégia ideal para Pedro quando } 0 \leqslant q \leqslant \frac{1}{3},} \\
& {[1 ; 0] \text { é uma contra-estratégia ideal para Pedro quando } \frac{1}{3} \leqslant q \leqslant 1 .}
\end{aligned}
$$

Em outras palavras, caso João não defenda mais que $\frac{1}{3}$ das vezes à esquerda, Pedro deve persistir com cobranças à esquerda. Uma vez que João pule mais que $\frac{1}{3}$ à esquerda, então, Pedro chutará sempre à direita. Finalmente, quando $q=\frac{1}{3}$ independente da estratégia de Pedro, o ganho será o mesmo.

Como a interseç̧ão dos gráficos de $h_{1}(q)$ e $h_{2}(q)$ também representa o ponto mais baixo de $G_{\mathcal{J}}$, isto quer dizer, que para esse valor de $q$ temos a escolha ideal para João. Impondo a estratégia a seguir:

$$
\left[1-\frac{1}{3} ; \frac{1}{3}\right]=\left[\frac{2}{3} ; \frac{1}{3}\right]
$$

o valor mínimo esperado por João é dado por 


$$
h_{1}\left(\frac{1}{3}\right)=0,8 \cdot \frac{1}{3}+0,2=\frac{8}{30}+0,2=0,26 \ldots+0,2=0,46 \ldots=46,6 \ldots \% .
$$

$\mathrm{Ou}$

$$
h_{2}\left(\frac{1}{3}\right)=-0,1 \cdot \frac{1}{3}+0,5=-\frac{1}{30}+0,5=-0,03 \ldots+0,5=0,46 \ldots=46,6 \ldots \% .
$$

Está claro que o ponto de mínimo da função representa uma estratégia significativa. Assim, como o ponto de máximo da função $G_{\mathcal{P}}$, o gráfico de $G_{\mathcal{J}}$ pode ter mais de um ponto de mínimo. Analogamente ao capítulo anterior podemos enunciar a definição/teorema

Teorema 6.1 Se $(x, y)$ é o ponto de mínimo do gráfico de $G_{\mathcal{J}}$, então,

$$
[1-x ; x] \text { é uma estratégia Minimax para João, e y é o ganho Minimax esperado. }
$$

Se João aplicar a estratégia Minimax $[1-x ; x]$, então, ele pode impedir, em média, que Pedro ganhe nada mais que y em cada jogada.

Assim como no Teorema 5.1, o nome Minimax faz referência à escolha mínima de João entre as maiores escolhas de Pedro, ou seja, representa o ponto mais baixo da curva de máximo.

\section{Soluções de Jogos Soma zero}

Nas seções 5 e 6 encontramos uma coincidência nos exemplos. As expectativas de ganho eram iguais quando as estratégias de Maximin e Minimax eram impostas. Tal coincidência acontece devido ao valor das expectativas não mudarem conforme as partidas são disputadas. Porém, as estratégias Minimax e Maximin possuem definições diferentes. Informalmente falando, a estratégia Maximin é um "base"para Pedro, enquanto a estratégia Minimax é um "teto"nos ganhos de Pedro, imposto por João.

A certeza da coincidência para jogos repetitivos de soma-zero é o teorema central da Teoria dos Jogos. Esse teorema será inicialmente discutido em um contexto de jogos $2 \times 2$ e reformulado para um caso mais geral, o qual será demonstrado no final desse capítulo.

Teorema 7.1 Para qualquer jogo de soma-zero $2 \times 2$ há um único número $v$ em que

i) a estratégia Maximin de Pedro garante uma expectativa de ganho de mínimo v;

ii) a estratégia Minimax de João garante uma expectativa de ganho a Pedro que não exceda $v$.

Os dois capítulos anteriores tiveram como objetivo encontrar métodos e soluções de jogos de soma-zero $2 \times 2$. Faremos agora algumas simplificações para agilizar as análises feitas anteriormente. Inicialmente, vamos classificar os jogos de soma-zero $2 \times 2$ em dois tipos.

- Jogos $2 \times 2$ Estritamente Determinados - Dizemos que um jogo é estrtitamente determinado se as estratégias Minimax e Maximin são puras. Essa classificação recebe este 
nome, uma vez que, cada jogador já sabe o que esperar de seu adversário, ou seja, uma estratégia pura.

- Jogos $2 \times 2$ Não Estritamente Determinados - são todos os outros jogos que possuem estratégias de Maximin e Minimax não puras, ou seja, são estratégias mistas.

Para entendermos melhor os jogos estritamente determinados precisamos do conceito Ponto de Sela de um jogo de soma-zero $m \times n$.

Definição 7.1 Dizemos que um elemento $a_{i j}$ de uma matriz $A m \times n$ é um ponto de sela da matriz $A$, se ele for simultaneamente um mínimo em sua linha e um máximo em sua coluna, isto é, se

$$
\begin{aligned}
& a_{i j} \leqslant a_{i l} \quad \text { para todo } \quad l=1, \ldots, n \quad e \\
& a_{i j} \geqslant a_{k j} \quad \text { para todo } \quad k=1, \ldots, m .
\end{aligned}
$$

Exemplo 7.1 A entrada de número 3 representa o ponto de sela do jogo

Tabela 18. Exemplo 7.1

\begin{tabular}{|l|l|}
\hline 1 & 0 \\
\hline 3 & 4 \\
\hline
\end{tabular}

Vamos demonstrar dois lemas, para jogos estritamente determinados, necessários para a demonstração do Teorema Minimax que enunciaremos mais tarde.

Lema 7.1 Pedro tem uma estratégia Maximin pura se, e somente se, a matriz do jogo $G$ tem um ponto de sela, e nesse caso o ganho do ponto de sela é igual valor Maximin.

Demonstração: Dado um jogo qualquer

Tabela 19. Lema 7.1

$$
\mathrm{G}=\begin{array}{|l|l|}
\hline a_{11} & a_{12} \\
\hline a_{21} & a_{22} \\
\hline
\end{array}
$$

vamos assumir sem perda de generalidade que $a_{11} \leqslant a_{12}$. Suponha inicialmente que Pedro tem uma estratégia Maximin pura. Lembremos que os segmentos $g_{1}(p)=(1-p) a_{11}+p a_{21} \mathrm{e}$ $g_{2}(p)=(1-p) a_{12}+p a_{22} \operatorname{com} 0 \leqslant p \leqslant 1$ estão entre nos pontos $\left(0, a_{11}\right)$ e $\left(1, a_{21}\right)$ para $g_{1}(p)$ e $\left(0, a_{12}\right)$ e $\left(1, a_{22}\right)$ para $g_{2}(p)$. Como a estratégia Maximin é dada pelo ponto de máximo do gráfico de $G_{P}(p)$ então podemos afirmar, de acordo com a suposição acima, que os segmentos não se interceptam em um ponto interior (Fig. 13.1 e 13.2), os segmentos possuem coeficientes angulares positivos (Fig. 13.3) ou ambos negativos (Fig. 13.4). Mantendo em mente ainda que $a_{11} \leqslant a_{12}$, podemos notar que, nos quatro casos possíveis, os pontos de sela que ocorrem em cada uma das figuras são dados por $a_{11}, a_{21}, a_{22}$ e $a_{11}$, respectivamente, e esses valores são iguais ao Maximin de cada jogo. 
Revista Ciências Exatas e Naturais, Vol.19, nº.2, Jul/Dez, 2017

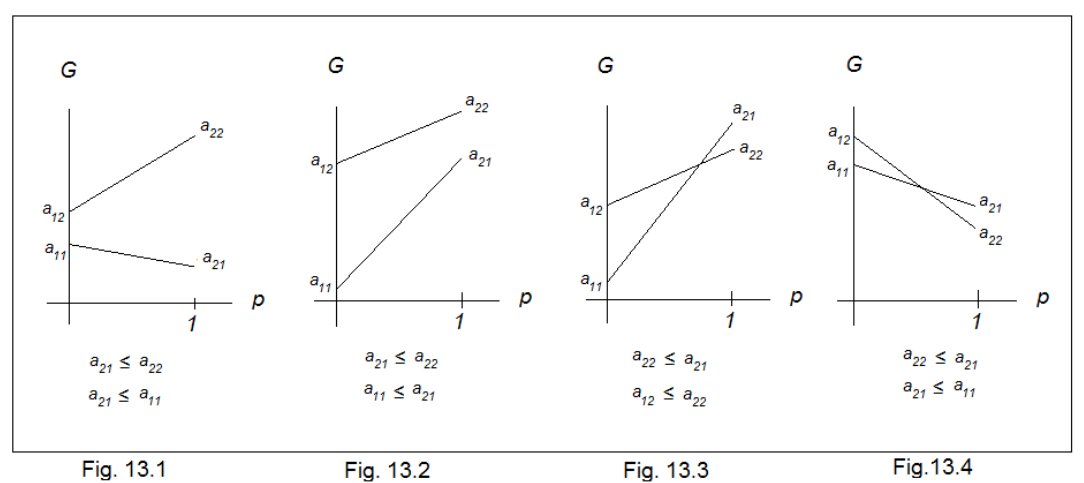

Figura 4. Demonstração - Lema 7.1

Suponha, agora, que o jogo $G$ admita um ponto de sela. Esse ponto de sela implica algumas desigualdades para o gráfico de $G_{P}(p)$ gerando assim quatro possibilidades para a função ganho de Pedro, considerando ainda a suposição que $a_{11} \leqslant a_{12}$, os casos são dados pelas figuras 14.1 a 14.4. Perceba facilmente, que em todos os casos, existe uma estratégia Maximin pura e o valor do ganho coincide com o ponto de sela.

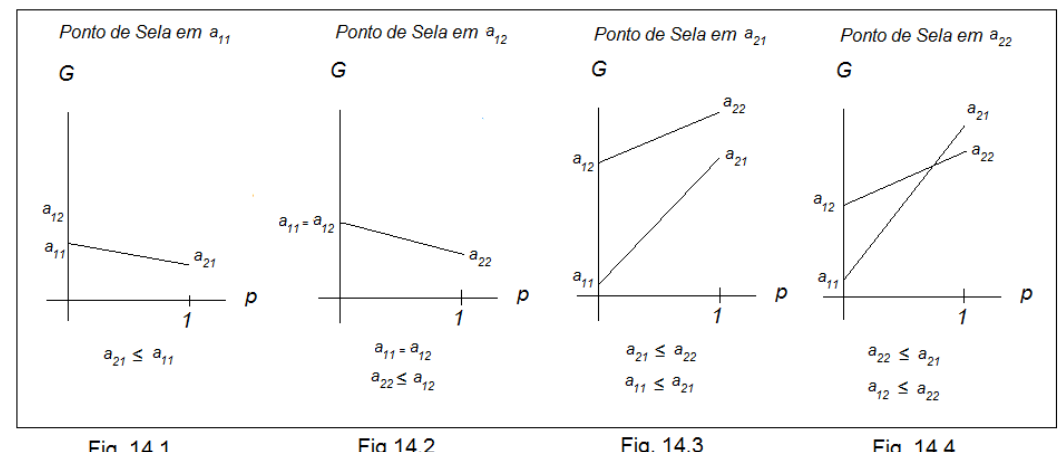

Figura 5. Demonstração - Lema 7.1

Lema 7.2 João tem uma estratégia Minimax pura se, e somente se, o jogo G tem um ponto de sela, e nesse caso o ganho do ponto de sela é igual valor Minimax.

A demonstração é análoga ao Lema 7.1

Os jogos não estritamente determinados são sujeitos a um procedimento de solução diferentes, porém tão simples quanto os estritamente determinados. Seja o jogo 
FRANCEZ, D. J.

Tabela 20. Jogo não determinado

$$
\begin{array}{cc|c|c|} 
& \multicolumn{2}{c}{\text { João }} \\
\multirow{3}{*}{\text { Pedro }} & \multicolumn{1}{c}{1-\mathrm{q}} & \mathrm{q} \\
\cline { 3 - 4 } & \mathrm{p} & a_{11} & a_{12} \\
\cline { 3 - 4 } & a_{21} & a_{22} \\
\cline { 3 - 4 } & & &
\end{array}
$$

Retomando as funções $g_{1}(p)$ e $g_{2}(p)$ deduzidas quando João aplica uma contra-estratégia ideal pura temos:

$$
\begin{aligned}
& g_{1}(p)=(1-p) \cdot 1 \cdot a_{11}+p \cdot 1 \cdot a_{21}=a_{11}(1-p)+a_{21} p=\left(a_{21}-a_{11}\right) p+a_{11} \\
& g_{2}(p)=(1-p) \cdot 1 \cdot a_{12}+p \cdot 1 \cdot a_{22}=a_{12}(1-p)+a_{22} p=\left(a_{22}-a_{12}\right) p+a_{12}
\end{aligned}
$$

Já sabemos que um jogo não estritamente determinado tem uma estratégia mista que representa o Maximin para Pedro e o Minimax para João. Assim, o valor de $p$ é intersecção dos segmentos $g_{1}(p)$ e $g_{2}(p)$. Para encontramos tal valor, basta resolvermos a equação abaixo:

$$
\begin{aligned}
g_{1}(p) & =g_{2}(p) \\
\left(a_{21}-a_{11}\right) p+a_{11} & =\left(a_{22}-a_{12}\right) p+a_{12} \\
\left(a_{21}-a_{11}-a_{22}+a_{12}\right) p & =a_{12}-a_{11} \\
p=\frac{a_{11}-a_{12}}{a_{11}-a_{12}-a_{21}+a_{22}} & \text { com } \quad a_{11}-a_{12}-a_{21}+a_{22} \neq 0
\end{aligned}
$$

Consequetemente, a estratégia Maximin mista $[1-p, p]$ para Pedro é dada por

$$
\left[\frac{a_{22}-a_{21}}{a_{11}-a_{12}-a_{21}+a_{22}}, \frac{a_{11}-a_{12}}{a_{11}-a_{12}-a_{21}+a_{22}}\right]
$$

Portanto, temos um método simples de encontrar a estratégia Maximin para Pedro.

Teorema 7.2 (Minimax) Para qualquer jogo de soma-zero $2 \times 2$ há um único número $v$ em que:

i) a estratégia Maximin de Pedro garante uma expectativa de ganho de minimo v;

ii) a estratégia Minimax de João garante uma expectativa de ganho a Pedro que não exceda $v$.

Demonstração: Caso o jogo venha a ter um ponto de sela, então o Teorema se resume no Lemas 7.1 e 7.2 . Caso o jogo não tenha um ponto de sela, ou seja, um jogo não estritamente determinado, podemos utilizar a teoria do Cálculo para demonstra-lo.

Suponha que em um jogo qualquer Pedro aplique a estratégia $[1-x ; x]$ e João $[1-y ; y]$. A situação se resume no quadro abaixo:

Tabela 21. Demonstração Teorema Minimax

\section{João}

\begin{tabular}{cc|c|c|}
\multirow{4}{*}{ Pedro } & \multicolumn{1}{c}{$1-\mathrm{y}$} & \multicolumn{1}{c}{$\mathrm{y}$} \\
\cline { 3 - 4 } & $\mathrm{x}$ & $a_{11}$ & $a_{12}$ \\
\cline { 3 - 4 } & $a_{21}$ & $a_{22}$ \\
\cline { 3 - 4 } & & &
\end{tabular}


Revista Ciências Exatas e Naturais, Vol.19, nº.2, Jul/Dez, 2017

Note então que a função ganho de Pedro é dada por:

$$
G_{\mathcal{P}}(x, y)=(1-x) \cdot(1-y) \cdot a_{11}+(1-x) \cdot y \cdot a_{12}+x \cdot(1-y) \cdot a_{21}+x \cdot y \cdot a_{22}
$$

Simplificando a função em termos de x e y, obtemos:

$$
G_{\mathcal{P}}(x, y)=x y\left(a_{11}-a_{12}-a_{21}+a_{22}\right)+x\left(a_{21}-a_{11}\right)+y\left(a_{12}-a_{11}\right)+a_{11}
$$

Eventualmente definida para $0 \leqslant x, y \leqslant 1$ uma vez que $[1-x, x]$ e $[1-y, y]$, são as respectivas estratégias aplicadas por Pedro e João.

Vamos agora encontrar um valor da função $G_{\mathcal{P}}(x, y)$ que seja máximo na direção $x$ e mínimo na direção $y$, ou seja,um ponto de sela de acordo com a teoria do Cálculo. É importante não confundir ponto de sela do cálculo, com o ponto de sela da teoria dos jogos, ambos são diferentes em suas definições. A figura abaixo mostra um ponto de sela do cálculo em uma curva dada.

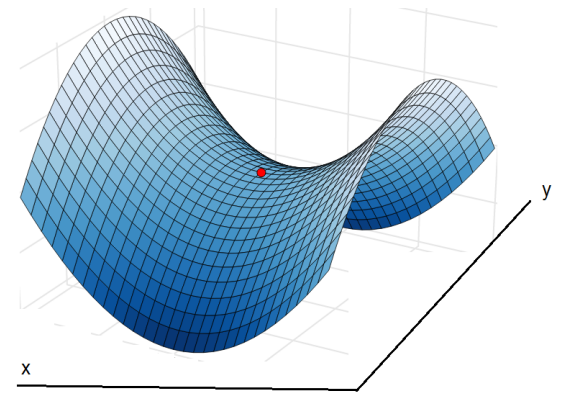

Figura 6. Ponto Sela Cálculo

Sabemos que o ponto de sela se dá em um dos seus pontos críticos, ou seja, nos pontos em que as derivadas parciais são nulas, isto é:

$$
\frac{\partial G_{\mathcal{P}}}{\partial x}=0 \quad \text { e } \quad \frac{\partial G_{\mathcal{P}}}{\partial y}=0 .
$$

Calculando as derivadas parciais temos:

$$
\begin{aligned}
& \frac{\partial G_{\mathcal{P}}}{\partial x}=y\left(a_{11}-a_{12}-a_{21}+a_{22}\right)+\left(a_{21}-a_{11}\right)=0, \\
& \frac{\partial G_{\mathcal{P}}}{\partial y}=x\left(a_{11}-a_{12}-a_{21}+a_{22}\right)+\left(a_{12}-a_{11}\right)=0 .
\end{aligned}
$$

Com isso concluímos que:

$$
y=\frac{a_{11}-a_{21}}{a_{11}-a_{12}-a_{21}+a_{22}} \quad \text { e } \quad x=\frac{a_{11}-a_{12}}{a_{11}-a_{12}-a_{21}+a_{22}}
$$

Tendo em mente que um jogo não estritamente determinado admite $a_{11}-a_{12}-a_{21}+a_{22} \neq$ 0 , pois caso contrário, o gráfico de $G_{\mathcal{P}}$ seria um plano e consequentemente teria a estratégia Maximin e Minimax em um de seus vértices, conforme visto anteriormente.

Portanto o ponto $\left(\frac{a_{11}-a_{12}}{a_{11}-a_{12}-a_{21}+a_{22}} ; \frac{a_{11}-a_{21}}{a_{11}-a_{12}-a_{21}+a_{22}}\right)$ é um ponto crítico de $G_{\mathcal{P}}$, classifiquemos agora o ponto crítico usando o teste da derivada segunda e a matriz Hessiana. 


$$
\Delta\left(x_{0}, y_{0}\right)=\operatorname{det}\left(\begin{array}{cc}
\frac{\partial^{2}}{\partial x^{2}} & \frac{\partial^{2}}{\partial x \partial y} \\
\frac{\partial^{2}}{\partial y \partial x} & \frac{\partial^{2}}{\partial y^{2}}
\end{array}\right)
$$

Calculando as derivadas segundas de $G_{\mathcal{P}}$ obtemos:

$$
\begin{aligned}
\frac{\partial^{2}}{\partial x^{2}} & =0, & \frac{\partial^{2}}{\partial x \partial y} & =a_{11}-a_{12}-a_{21}+a_{22} \\
\frac{\partial^{2}}{\partial y^{2}} & =0, & \frac{\partial^{2}}{\partial y \partial x} & =a_{11}-a_{12}-a_{21}+a_{22} .
\end{aligned}
$$

Substituindo os valores acima na matriz Hessiana e calculando o determinante temos:

$$
\begin{aligned}
& \Delta\left(x_{0}, y_{0}\right)=\operatorname{det}\left(\begin{array}{cc}
0 & a_{11}-a_{12}-a_{21}+a_{22} \\
a_{11}-a_{12}-a_{21}+a_{22} & 0
\end{array}\right) \\
& \Delta\left(x_{0}, y_{0}\right)=-\left(a_{11}-a_{12}-a_{21}+a_{22}\right)^{2}
\end{aligned}
$$

Com isso, mostramos que o determinante da matriz Hessiana é negativo para todo valor de $x$ e $y$. Assim o ponto de sela

$$
\left(\frac{a_{11}-a_{12}}{a_{11}-a_{12}-a_{21}+a_{22}} ; \frac{a_{11}-a_{21}}{a_{11}-a_{12}-a_{21}+a_{22}}\right)
$$

representa o máximo na direção $x$ e mínimo na direção $y$, ou vice-versa, portanto

$$
v=G_{\mathcal{P}}\left(\frac{a_{11}-a_{12}}{a_{11}-a_{12}-a_{21}+a_{22}} ; \frac{a_{11}-a_{21}}{a_{11}-a_{12}-a_{21}+a_{22}}\right)
$$

representa o Maximin para Pedro e o para João Minimax.

Ao analisar o Teorema Minimax, observamos que a função $G_{\mathcal{P}}(p, q)$ sempre possuirá um ponto de sela do cálculo, desde que a condição $a_{11}-a_{12}-a_{21}+a_{22} \neq 0$ seja satisfeita. Contudo, o ponto de sela pode estar dentro do domínio da função ou não. Caso esteja dentro, o jogo será não estritamente determinado e consequentemente o ponto de sela representa a estratégia Maximin, Minimax e o valor esperado do ganho. Por outro lado, se o ponto o ponto de sela estiver fora do domínio, ou $a_{11}-a_{12}-a_{21}+a_{22}=0$, o que representaria um plano, então, o jogo será estritamente determinado. Assim, a estratégia Minimax e Maximin será dada por um dos vértices do gráfico de $G_{\mathcal{P}}(p, q)$.

Em resumo, a função $G_{\mathcal{P}}(p, q)$ :

- Representa um jogo estritamente determinado se:

i) Se a função representa um plano, ou seja, $a_{11}-a_{12}-a_{21}+a_{22}=0$;

ii) Se o ponto de sela não pertencer ao domínio da função;

- Representa um jogo não estritamente determinado se:

i) Se o ponto de sela pertencer ao quadrado $[0,1] \times[0,1]$, ou seja, pertencer ao domínio da função. 
Revista Ciências Exatas e Naturais, Vol.19, nº.2, Jul/Dez, 2017

\section{Resultados e discussão}

Ao propor utilizar jogos de estratégia para analisar quaisquer situações de conflito, muitos matemáticos retornaram a uma prática milenar para entender e estudar o mundo. Ao fazer isso, criaram uma ciência com uma grande capacidade de generalização e precisão matemática.

Em uma breve discussão sobre alguns jogos, percebemos que um jogador pode obter alguma vantagem sobre seu oponente, dependendo de suas escolhas. Com isso, definimos o importante conceito de estratégia, bem como sua classificação em: estratégia pura e estratégia mista. Concluímos que a melhor contra estratégia, ou seja, a estratégia que gera um maior ganho é necessariamente a pura.

Nas seções quatro e cinco mostramos que cada jogador tem uma estratégia ideal, pelo menos do ponto de vista defensivo. Tais estratégias são denominadas: Maximin e Minimax. Ao aplicar a estratégia Maximin obtém-se o ganho máximo, quando o oponente tenta minimizá-lo, ou seja, um valor 'base'. Por outro lado, a estratégia Minimax gera o ganho mínimo do oponente, quando o mesmo tenta maximizá-lo, ou seja, um 'teto'.

Ao fim do trabalho, mostramos que os jogos de soma-zero $2 \times 2$ podem ser classificados em jogos estritamente determinados e não estritamente determinados. Definimos nesse momento, o importante conceito de ponto de sela e mostramos, formalmente, que todo jogo estritamente determinado o possui, e que as estratégias que representam o Maximin e Minimax são puras. Já em jogos não estritamente determinados, mostramos que as estratégias ideais são mistas. Tal afirmação é demonstrada pelo Teorema Minimax.

\section{Referências}

[1] CONWAY, J. H. All games bright and beautiful. American Mathematical Monthly, p. 417-434, 1977.

[2] DUTTA, P. K. Strategies and games: theory and practice. MIT press, 1999.

[3] FIANI, R. Teoria dos jogos. Elsevier Brasil, 2006.

[4] FUDEnBERG, D.; TIROLE, J. Game theory, 1991. Cambridge, Massachusetts, v. 393, p. 12, 1991.

[5] GUIDORIZZI, H. L. Um curso de Cálculo, vol. 2. Grupo Gen-LTC, 2000.

[6] OSBORNE, M. J.; RUBINSTEIN, A. A course in game theory. MIT press, 1994.

[7] RASMUSEN, E.; BLACKWELL, B. Games and information. Cambridge, MA, v. 15, 1994

[8] SCHELLING, Thomas C. The strategy of conflict. Harvard university press, 1980.

[9] SARTINI, B. A. et al. Uma introdução à teoria dos jogos. II Bienal da SBM?Universidade Federal da Bahia, p. 1-61, 2004.

[10] VON NEUMANN, J.; MORGENSTERN, O. Theory of games and economic behavior. Princeton university press, 2007. 